\title{
HIP SURGERY
}

\section{Decision making on timing of surgery for hip fracture patients on clopidogrel}

\author{
B Purushothaman ${ }^{1}$, M Webb ${ }^{2}$, A Weusten ${ }^{3}$, S Bonczek ${ }^{2}$, J Ramaskandhan $^{4}$, A Nanu ${ }^{1}$ \\ ${ }^{1}$ City Hospitals Sunderland NHS Foundation Trust, UK \\ ${ }^{2}$ South Tees Hospitals NHS Foundation Trust, UK \\ ${ }^{3}$ Gateshead Health NHS Foundation Trust, UK \\ ${ }^{4}$ Newcastle upon Tyne Hospitals NHS Foundation Trust, UK
}

\section{ABSTRACT}

Patients taking clopidogrel who sustain a fractured neck of femur pose a challenge to orthopaedic surgeons. The aim of this study was to determine whether delay to theatre for these patients affects drop in haemoglobin levels, need for blood transfusion, length of hospital stay and 30-day mortality. A retrospective review of all neck of femur patients admitted at two centres in the North East of England over 3 years revealed 85 patients.

Patients were divided into two groups depending on whether they were taking clopidogrel alone (C) or with aspirin (CA). Haemoglobin drop was significantly different in the CA group that was operated on early (CA1) versus the group for which surgery was delayed by over 48 hours (CA2): $3.3 \mathrm{~g} / \mathrm{dl}$ and $1.9 \mathrm{~g} / \mathrm{dl}$ respectively $(p=0.01)$. The mean inpatient stay in group $\mathrm{C}$ was 35.9 days while in group CA it was 19.9 days $(p=0.002$ ). The mean length of stay in group CA2 (26.7 days) was significantly longer than for CA1 patients (14.1 days) ( $p=0.01$ ). There were no significant differences in mortality or wound complications.

Hip fracture patients on clopidogrel can be safely operated on early provided they are medically stable. Bleeding risk should be borne in mind in those patients on dual therapy with aspirin.

\section{KEYWORDS}

Hip fracture - Clopidogrel - Aspirin - Delay to surgery

Accepted 28 June 2015

CORRESPONDENCE TO

Mark Webb, E: markwebb@nhs.net

Clopidogrel is a thienopyridine that irreversibly binds to platelets inhibiting aggregation. It is used commonly with or without aspirin for primary and secondary prevention of cardio and cerebrovascular disease. Given the prevalence of coronary disease, advances in coronary intervention for stable and unstable cardiac as well as stroke disease, it is no surprise that the number of patients admitted to hospital on dual antiplatelet therapy (DAPT) as well as other newer anticoagulant agents is rising. The timing of operative intervention for hip fracture patients on antiplatelet agents poses a significant challenge to the multidisciplinary team managing these complex patients. ${ }^{1}$

Hip fracture patients tend to be older with co-morbidities. There is consensus in the orthopaedic community that if feasible and indicated, surgery should be carried out at the earliest opportunity followed by early mobilisation. Patients taking DAPT, clopidogrel alone or other anticoagulants have an elevated bleeding risk in the perioperative period. These patients have a higher risk of bleeding if operated on early while delaying surgery is associated with increased risk of morbidity and mortality. ${ }^{2}$

There is a lack of consensus among orthopaedic teams for timing of operation, with some surgeons choosing to stop their patients' antiplatelet treatment and delay operative management. ${ }^{3,4}$ There is a paucity of literature reporting the risks and benefits of operating on these patients. This study sought to determine whether time to surgery influenced factors including drop in haemoglobin $(\mathrm{Hb})$ levels, need for blood transfusion, duration of inpatient stay and 30-day mortality for patients who were admitted with a neck of femur fracture while taking clopidogrel.

\section{Methods}

This was a retrospective cohort study conducted across two National Health Service trust hospitals (Hospital A and Hospital B) in the North East of England. It was registered with local clinical governance departments at both hospitals for clinical audit purposes and so ethical approval was not required. The hospital hip fracture database was used to track all patients admitted with hip fractures at Hospital A (January 2007 - March 2011) and Hospital B (March 2009 - March 2011). Patient notes were screened to identify hip fracture patients taking clopidogrel as a regular medication prior to sustaining a fractured neck of femur. 
Patient demographics included age, sex and medical comorbidities. Inpatient data extracted included past medical history, type of fracture, date of admission, date of surgery, type of anaesthesia, ASA (American Society of Anesthesiologists) grade, blood transfusion, indication for clopidogrel administration and history of dual therapy with aspirin. The Charlson co-morbidity index (CCI) was calculated. Laboratory investigation details collected included pre and postoperative $\mathrm{Hb}$ levels. Information on postoperative complications was extracted along with date of discharge and duration of stay for all patients. Patients were divided into two groups: C (clopidogrel only) and CA (clopidogrel and aspirin). These groups were further subdivided into two subgroups based on the time to surgery (early [ $<48$ hours]: groups C1 and CA1, late [>48 hours]: groups C2 and CA2).

\section{Statistical analysis}

Statistical analysis was performed with SPSS $^{\circledR}$ version 17 (SPSS, Chicago, IL, US). Demographic factors were compared using Student's t-test and chi-squared analysis as appropriate. Univariate analysis using the general linear model was performed for comparison of outcomes (drop in $\mathrm{Hb}$, duration of inpatient stay). This was modelled to adjust for age, which was different between the groups and could have an influence on outcomes as an independent predictor. The 30-day mortality was compared with a chi-squared test.

\section{Results}

Eighty-five patients who were on clopidogrel and sustained a hip fracture were admitted during the study period. Fourteen patients were excluded from the study (4 did not have surgery as they were medically unfit and 10 had either intraoperative blood transfusion or immediate postoperative transfusion). Of the remaining 71 patients, 43 were admitted on clopidogrel only (group C) while 28 were taking both clopidogrel and aspirin (group CA). The groups were further subdivided into group 1 (early surgery, <48 hours) and group 2 (delayed surgery, $>48$ hours). There were 23 patients $(53.5 \%)$ in group C1 and $20(46.5 \%)$ in group C2. In group CA1, there were 15 patients (53.4\%) and $13(46.4 \%)$ in group CA2 (Table 1).

The mean age of patients in groups $\mathrm{C}$ and CA was 83.2 years (standard deviation [SD]: 8.2 years, range: $66-100$ years) and 80.8 years (SD: 12.6 years, range: 51-100 years) respectively. There was no significant difference in age between the cohorts $(p=0.33)$. However, when comparing subgroups $\mathrm{C} 1$ and $\mathrm{C} 2$, patients in the former subgroup were significantly older (mean: 87.3 years, SD: 7.7 years) than those in the latter (mean: 78.7 years, SD: 6.4 years) $(p<0.001)$. In group CA, there was no significant differences in age between subgroups CA1 (mean: 81.3 years, SD: 13.9 years) and CA2 (mean: 80.3 years, SD: 11.4 years) $(p=0.84)$.

The male-to-female ratios in groups $C$ and CA were 9:34 and 8:20 respectively. In terms of subgroups, there was no significant difference in the ratios for subgroups C1 and C2 (5:19 vs $5: 16, p=0.81)$. Similar results were seen in subgroups CA1 and CA2 (6:9 vs 2:11, $p=0.13$ ). The number of co-morbidities reported by CA patients (mean: 3.6, SD: 1.2) was significantly higher than in group $\mathrm{C}$ (mean: 2.9, SD: 1.5) $(p=0.05)$. There was no significant difference in comorbidities reported between subgroups $\mathrm{C} 1$ and $\mathrm{C} 2$ (2.6 vs 3.3, $p=0.14$ ) or between subgroups CA1 and CA2 (3.3 vs 3.6, $p=0.85$ ).

\section{Indication for antiplatelet agents}

Cardiac disease was the most common indication for both groups C $(67.4 \%)$ and CA $(78.6 \%)$. One patient in each cohort was on clopidogrel following coronary stenting. Just over a fifth $(20.9 \%)$ of patients in group C were on

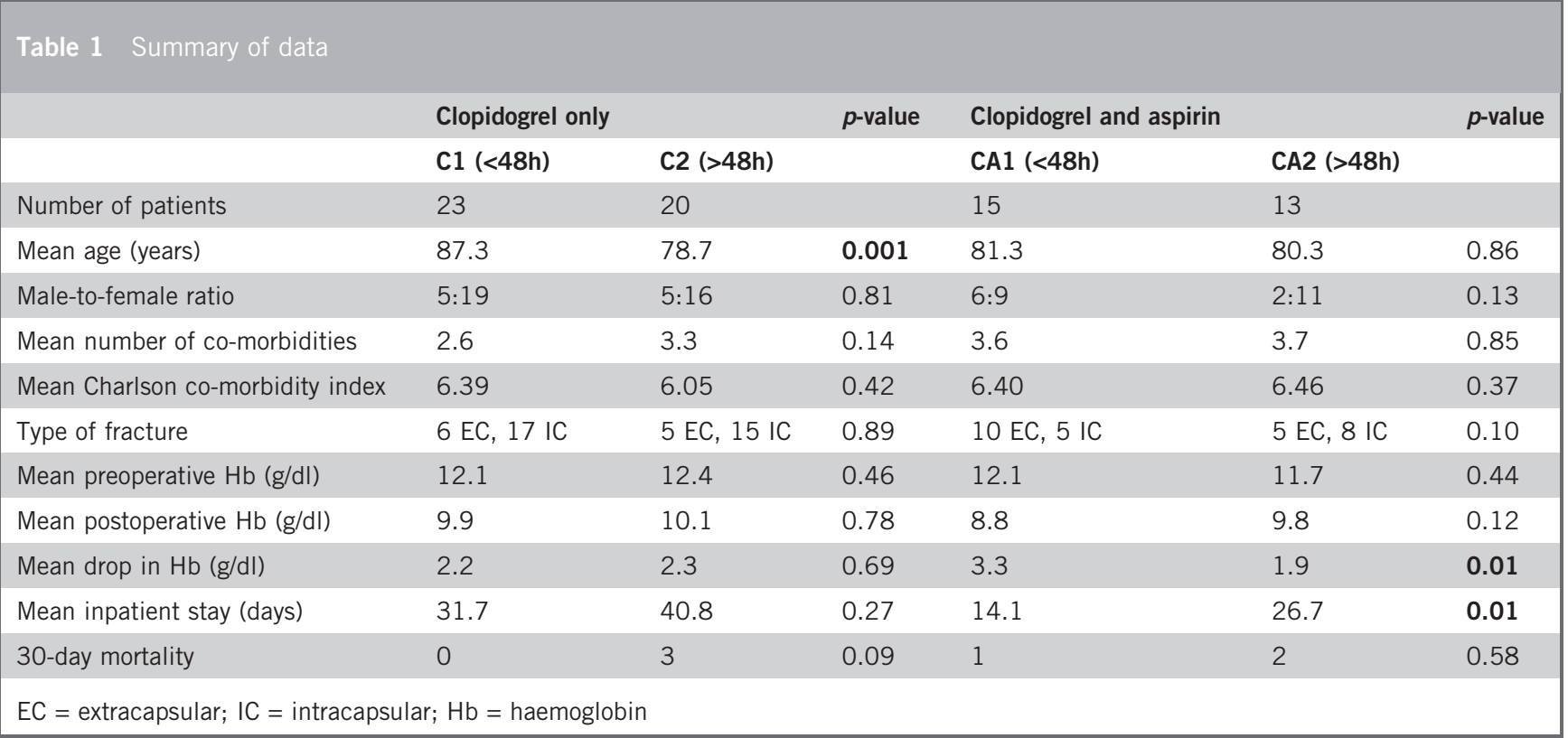


antiplatelet medication for prevention of cerebrovascular accidents. For CA patients, the proportion was slightly lower $(14.3 \%)$. Seven per cent of patients in each cohort were on antiplatelet medication for atrial fibrillation.

\section{Charlson co-morbidity index}

The mean CCI in group C was 6.23 (SD: 2.03); for CA patients, it was 6.43 (SD: 1.47). This difference was not statistically significant $(p=0.25)$. In group C, the CCI in subgroup $\mathrm{C} 1$ was 6.39 and for $\mathrm{C} 2$ patients, it was 6.05 . In subgroup CA1, it was 6.40 and in subgroup CA2, it was 6.46.

\section{Type of fracture and treatment methods}

There were 48 extracapsular and 23 intracapsular fractures. There was a relatively equal distribution of intracapsular ( $n=13)$ and extracapsular $(n=15)$ fractures in group CA while for patients in group C, the intracapsular fractures $(n=32)$ were three times the number of extracapsular fractures $(n=11)$. Of the 48 extracapsular fractures, 44 were treated with dynamic hip screw fixation and 4 were operated for with intramedullary hip screws. Of the 23 intracapsular fractures, 2 patients underwent a total hip arthroplasty while the rest underwent a cemented Thompson hemiarthroplasty. In the early operative subgroups (C1 and CA1), the majority had general anaesthesia for their surgery $(95.7 \%$ and $86.7 \%$ respectively) while in the delayed operative subgroups (C2 and CA2), nearly twothirds of the patients underwent general anaesthesia (60.5\% and $61.5 \%$ respectively).

\section{Drop in haemoglobin}

The mean preoperative $\mathrm{Hb}$ level in group $\mathrm{C}$ was $12.2 \mathrm{~g} / \mathrm{dl}$ (SD: $1.2 \mathrm{~g} / \mathrm{dl}$, range: $9.7-16.1 \mathrm{~g} / \mathrm{dl}$ ) and for CA patients, it was $11.9 \mathrm{~g} / \mathrm{dl}$ (SD: $1.4 \mathrm{~g} / \mathrm{dl}$, range: $9.3-15.1 \mathrm{~g} / \mathrm{dl}) \quad(p=0.40)$. The mean preoperative $\mathrm{Hb}$ levels in subgroups $\mathrm{C} 1$ and $\mathrm{C} 2$ were $12.1 \mathrm{~g} / \mathrm{dl}$ and $12.4 \mathrm{~g} / \mathrm{dl}$ respectively $(p=0.46)$. In subgroups CA1 and CA2, they were $12.1 \mathrm{~g} / \mathrm{dl}$ and $11.7 \mathrm{~g} / \mathrm{dl}$ respectively $(p=0.44)$.

The mean postoperative $\mathrm{Hb}$ level in group $\mathrm{C}$ was $9.9 \mathrm{~g} / \mathrm{dl}$ (SD: $1.4 \mathrm{~g} / \mathrm{dl}$, range: $6-12.1 \mathrm{~g} / \mathrm{dl}$ ) and in group CA, it was $9.2 \mathrm{~g} / \mathrm{dl}$ (SD: $1.6 \mathrm{~g} / \mathrm{dl}$, range: $5.8-13.3 \mathrm{~g} / \mathrm{dl}) \quad(p=0.058)$. The mean postoperative $\mathrm{Hb}$ levels for $\mathrm{C} 1$ and $\mathrm{C} 2$ patients were $9.9 \mathrm{~g} / \mathrm{dl}$ and $10.1 \mathrm{~g} / \mathrm{dl}$ respectively $(p=0.78)$. In subgroups CA1 and CA2, they were $8.8 \mathrm{~g} / \mathrm{dl}$ and $9.8 \mathrm{~g} / \mathrm{dl}$ respectively $(p=0.12)$. The drop in $\mathrm{Hb}$ in group $\mathrm{C}$ was $2.2 \mathrm{~g} / \mathrm{dl}$ while for CA patients, it was $2.7 \mathrm{~g} / \mathrm{dl}(p=0.20)$, subgroups $\mathrm{C} 1$ being $2.2 \mathrm{~g} / \mathrm{dl}$ and $\mathrm{C} 22.3 \mathrm{~g} / \mathrm{dl}(p=0.69)$. However, the drop in $\mathrm{Hb}$ was significantly different between subgroups CA1 and CA2 $(3.3 \mathrm{~g} / \mathrm{dl}$ and $1.9 \mathrm{~g} / \mathrm{dl}$ respectively, $p=0.01)$.

\section{Transfusion}

Four patients (17.4\%) in subgroup C1 and two C2 patients $(10.0 \%)$ needed a transfusion. Five patients $(33.4 \%)$ in subgroup CA1 needed a total of 12 units and four (30.8\%) in subgroup CA2 had a total of seven units.

\section{Inpatient stay}

The mean length of stay in group $\mathrm{C}$ was 35.9 days (SD: 26.0 days, range: 7-136 days) while CA patients had a comparatively shorter stay of 19.9 days (SD: 13.7 days, range: 5-63 days) $(p=0.002)$. When comparing the subgroups in group C, the length of stay for C1 patients was 31.7 days (SD: 22.1 days, range: 7-78 days) while subgroup C2 had a slightly longer stay of 40.8 days (SD: 31.3 days, range: $8-136$ days). This was not statistically significant $(p=0.27)$. The mean length of stay in subgroup CA2 (26.7 days, SD: 10.3 days, range: 15-46 days) was significantly longer than for CA1 patients (14.1 days, SD: 14.9 days, range: $5-63$ days) ( $p=0.01)$.

\section{Complications}

Three patients had thromboembolic complications. Two patients were in group C and one C1 patient had pulmonary embolism. One patient in subgroup $\mathrm{C} 2$ had deep vein thrombosis and one CA2 patient had a cerebrovascular event. None of the patients had to return to theatre for surgical wound related problems.

\section{Thirty-day mortality}

In group C, the 30-day mortality rate was 9.3\% (4/43) and all of the patients who died were in subgroup C2 $(p=0.09)$. The 30-day mortality rate in group CA was $10.7 \%(3 / 28)$. Two of these cases were in subgroup CA2 and one was in subgroup CA1 $(p=0.58)$.

\section{Discussion}

Clopidogrel, an increasingly used antiplatelet drug, is a thienopyridine derivative and acts by irreversibly inhibiting adenosine diphosphate (ADP) induced platelet aggregation. Inhibition of platelet aggregation to ADP by clopidogrel is highly variable and shows a normal distribution, with an average of $40-50 \%$ inhibition. $^{7}$ Platelets once exposed to clopidogrel are therefore affected for their lifetime of 7-10 days. ${ }^{8}$ Aspirin irreversibly blocks the formation of thromboxane $\mathrm{A} 2$ in platelets and inhibits platelet aggregation through inhibition of the cyclooxygenase-1 enzyme. ${ }^{9}$

The National Institute for Health and Care Excellence recommends lifelong prescription of clopidogrel for the prevention of occlusive vascular events (including the treatment of cerebrovascular accidents, peripheral vascular disease, non-ST segment elevation acute coronary syndrome, patients with less tolerance to aspirin) and atherothrombosis following myocardial infarction. ${ }^{10}$ Clopidogrel combined with aspirin is of proven benefit in the secondary prevention of cardiovascular and cerebrovascular events. ${ }^{11}$ It is also the prophylactic regimen in patients with coronary stents. ${ }^{12,13}$

A Cochrane review in 2011 concluded that the use of clopidogrel plus aspirin is associated with a reduction in the risk of cardiovascular events and an increased risk of bleeding compared with aspirin alone. ${ }^{14}$ Only in patients with acute non-ST coronary syndrome do the benefits outweigh the harm. Furthermore, a randomised, open label, blinded endpoint trial (the CLAIR study) has shown that combination therapy is more effective than aspirin alone in reducing microembolic signals in patients with predominantly intracranial symptomatic stenosis. ${ }^{15}$ 
These studies suggest that the use of DAPT will increase exponentially. This may pose a significant challenge to the trauma team in years to come.

Surveys of orthopaedic departments in the UK have revealed a wide variation in practice regarding the discontinuation of clopidogrel preoperatively in patients due to undergo surgery for femoral neck fractures. ${ }^{3,4}$

Wilhite et al studied the effect of clopidogrel, aspirin and combination therapy on bleeding time in patients with peripheral arterial disease. ${ }^{16}$ They concluded that aspirin and clopidogrel together had a more pronounced effect on bleeding time $(17.39 \pm 4.59$ minutes $)$ than just aspirin $(6.64$ \pm 3.52 minutes $)$ or just clopidogrel $(10.17 \pm 5.4$ minutes $)$ $(p<0.01)$

Conversely, Stone et al reviewed patients undergoing peripheral arterial surgery in whom clopidogrel was continued either alone or as part of DAPT. ${ }^{17}$ These patients did not have significant bleeding complications compared with patients taking no antiplatelet therapy or aspirin alone at the time of surgery. This suggests that clopidogrel can safely be continued in patients with appropriate indications for its use, such as symptomatic carotid disease or recent drug eluting coronary stents.

Intraoperative blood loss, perioperative blood loss and the amount of transfused blood in patients on antiplatelet drugs undergoing hip fracture surgery was compared with matched controls by Chechik et al. ${ }^{18}$ They had 22 patients on aspirin, 29 patients on clopidogrel, and 15 patients on clopidogrel and aspirin. They reported a mean perioperative blood loss of $900 \mathrm{ml}$ in the control and aspirin only groups, $1,091 \mathrm{ml}$ for patients on clopidogrel, and 1,312ml for those on clopidogrel and aspirin. The transfusion rate was 1.38 units in the clopidogrel only group, and 2.13 in the clopidogrel and aspirin group. The mean time to surgery was $51 \pm 25$ hours for the clopidogrel group, and 77 \pm 48 hours for the clopidogrel and aspirin group.

Chechik et al found that the drop in $\mathrm{Hb}$ was comparable in both the early and delayed surgery groups of patients on clopidogrel alone. ${ }^{18}$ However, there was a statistically significant drop in $\mathrm{Hb}$ in the early surgery group compared with the delayed group of patients on clopidogrel and aspirin $(3.3 \mathrm{~g} / \mathrm{dl}$ vs $1.9 \mathrm{~g} / \mathrm{dl}, p=0.01)$. Despite the increased $\mathrm{Hb}$ drop, the number of units transfused was not different between the early and delayed groups of patients on clopidogrel and aspirin.

In another paper, Chechik et al compared 30 patients who were on clopidogrel and underwent early surgery (mean time: 1 day and 16 hours, SD: 1 day) with a group of patients who underwent delayed surgery (mean time: 7 days and 12 hours, SD: 2 days and 17 hours). ${ }^{19}$ They found that the early treatment group had a shorter hospital stay than the delayed group (11 vs 17 days, $p=0.0002$ ). Even though the mortality rates were similar, there were more complications related to prolonged immobilisation in the delayed treatment group.

In our study, the length of hospital stay was longer in the clopidogrel only group than in the clopidogrel and aspirin group (35.9 vs 19.9 days). Among the patients on clopidogrel alone, the length of stay in the early surgery group was shorter (31.7 days) than for the delayed group (40.8 days) although this difference was not statistically significant. In the clopidogrel and aspirin group, there was a significant difference in the length of stay between the early (14.1 days) and delayed surgery group (26.7 days).

Leonidou et al reviewed 27 hip fracture patients on clopidogrel, whose mean time to theatre was 8 days. ${ }^{20}$ They found that the rates of medical complications following admission $(25.9 \%)$ and mortality $(14.8 \%)$ were higher than those from the National Hip Fracture Database (18\% and $11 \%$ respectively). However, this was not statistically significant. In our study, the 30-day mortality rate of patients on clopidogrel only was $9.3 \%$ versus $10.7 \%$ for patients on clopidogrel and aspirin. All clopidogrel only patients who died within 30 days were in the delayed treatment group, emphasising the importance of early surgery. The 30-day mortality for patients on clopidogrel and aspirin was not different between the early and delayed surgery groups.

Guidelines suggest discontinuing aspirin for at least 12 hours if taken for secondary prevention and 3 days if taken for primary prevention, and discontinuing clopidogrel for at least 5 days owing to the risk of spinal haematomas. ${ }^{21}$ Interestingly, in our study, one C1 patient and two CA1 patients ( $<48$ hours) had undergone spinal anaesthesia for their surgery. They did not develop a spinal haematoma or related complications. This is similar to the findings in a case series of vascular surgery patients on clopidogrel undergoing epidural anaesthesia. ${ }^{22}$ Furthermore, Collyer et al stated that no haematomas were reported for 25 hip fracture patients on clopidogrel who had spinal anaesthesia. ${ }^{23}$

The plasma half-life of clopidogrel is approximately six hours. ${ }^{24}$ Its concentration falls to $<2 \%$ after 24 hours. ${ }^{25}$ It is therefore safe to perform surgery 24 hours after the last dose. If platelets need to be transfused, they will not be affected significantly by the clopidogrel already present in circulation. Based on our observations, we propose that patients on clopidogrel as monotherapy or dual therapy with aspirin can be operated on safely after 24 hours of the last dose. The bleeding risk for patients on dual therapy should be considered. Ideally, an experienced surgeon would undertake the procedure, taking adequate measures to achieve haemostasis.

\section{Study limitations}

The retrospective design of the study meant that wound related complications treated in the community might have been missed. There were not many patients on clopidogrel following coronary stenting. While managing this group of patients, a multidisciplinary approach involving the cardiologists and anaesthetists should be followed. The number of patients in the study was small. Nevertheless, it is one of the largest series reported to date. The percentage of hip fracture patients on antiplatelet medication in our study (approximately $3 \%$ ) is comparable with others. ${ }^{25}$

Owing to the small number of patients on antiplatelet medications, data had to be collected over three years. However, the number of such patients is likely to rise in the future. 


\section{Conclusions}

Hip fracture patients on clopidogrel can be safely operated on early provided they are medically stable. Bleeding risk should be borne in mind in those patients on dual therapy with aspirin.

\section{References}

1. Johansen A, White J, Turk A. Clopidogrel therapy - implications for hip fracture surgery. Injury 2008; 39: 1,188-1,190.

2. Lavelle WF, Demers Lavelle EA, Uhl R. Operative delay for orthopedic patients on clopidogrel (Plavix): a complete lack of consensus. J Trauma 2008; 64: 996-1,000.

3. Inman DS, Michla Y, Partington PF. Perioperative management of trauma patients admitted on clopidogrel (Plavix). A survey of orthopaedic departments across the United Kingdom. Injury 2007; 38: 625-630.

4. Palan J, Odutola A, White SP. Is clopidogrel stopped prior to hip fracture surgery - a survey of current practice in the United Kingdom. Injury 2007; 38 : 1,279-1,285.

5. Chechik $\mathrm{O}$, Amar E, Khashan $\mathrm{M}$ et al. In support of early surgery for hip fractures sustained by elderly patients taking clopidogrel: a retrospective study. Drugs Aging 2012; 29: 63-68.

6. Sim W, Gonski PN. The management of patients with hip fractures who are taking Clopidogrel. Australas J Ageing 2009; 28: 194-197.

7. Serebruany VL, Steinhubl SR, Berger PB et al. Variability in platelet responsiveness to clopidogrel among 544 individuals. J Am Coll Cardiol 2005; 45: 246-251.

8. Dineen PF, Curtin RJ, Harty JA. A review of the use of common antiplatelet agents in orthopaedic practice. J Bone Joint Surg Br 2010; 92: 1,186-1,191.

9. Vane JR. Inhibition of prostaglandin synthesis as a mechanism of action for aspirin-like drugs. Nat New Biol 1971; 231: 232-235.

10. National Institute for Health and Clinical Excellence. Clopidogrel and Modifiedrelease Dipyridamole for the Prevention of Occlusive Vascular Events. London: NICE; 2010.

11. Steinhubl SR, Berger PB, Mann JT et al. Early and sustained dual oral antiplatelet therapy following percutaneous coronary intervention. JAMA 2002; 288: 2,411-2,420.

12. Dawkins KD, Gershlick T, de Belder M et al. Percutaneous coronary intervention: recommendations for good practice and training. Heart 2005; 91 (Suppl 6): vi1-vi27.
13. Mehta SR, Yusuf S, Peters RJ et al. Effects of pretreatment with clopidogrel and aspirin followed by long-term therapy in patients undergoing percutaneous coronary intervention: the PCI-CURE study. Lancet 2001; 358: 527-533.

14. Squizzato A, Keller T, Romualdi E, Middeldorp S. Clopidogrel plus aspirin versus aspirin alone for preventing cardiovascular disease. Cochrane Database Syst Rev 2011; 1: CD005158.

15. Wong KS, Chen C, Fu J et al. Clopidogrel plus aspirin versus aspirin alone for reducing embolisation in patients with acute symptomatic cerebral or carotid artery stenosis (CLAIR study): a randomised, open-label, blinded-endpoint trial. Lancet Neurol 2010; 9: 489-497.

16. Wilhite DB, Comerota AJ, Schmieder FA et al. Managing PAD with multiple platelet inhibitors: the effect of combination therapy on bleeding time. $J$ Vasc Surg 2003; 38: 710-713

17. Stone DH, Goodney PP, Schanzer A et al. Clopidogrel is not associated with major bleeding complications during peripheral arterial surgery. J Vasc Surg 2011; 54: 779-784.

18. Chechik $\mathrm{O}$, Thein R, Fichman $\mathrm{G}$ et al. The effect of clopidogrel and aspirin on blood loss in hip fracture surgery. Injury 2011; 42: 1,277-1,282.

19. Chechik O, Amar E, Khashan M et al. In support of early surgery for hip fractures sustained by elderly patients taking clopidogrel. Drugs Aging 2012; 29: 63-68.

20. Leonidou A, Cam NB, Chambers IR. Femoral neck fractures in patients on Clopidogrel. The effect of delaying surgery and the introduction of the new SIGN guidelines. Surgeon 2011; 9: 318-321.

21. Breivik $\mathrm{H}$, Bang $\mathrm{U}$, Jalonen $\mathrm{J}$ et al. Nordic guidelines for neuraxial blocks in disturbed haemostasis from the Scandinavian Society of Anaesthesiology and Intensive Care Medicine. Acta Anaesthesiol Scand 2010; 54: 16-41.

22. Osta WA, Akbary $\mathrm{H}$, Fuleihan SF. Epidural analgesia in vascular surgery patients actively taking clopidogrel. Br J Anaesth 2010; 104: 429-432.

23. Collyer TC, Reynolds HC, Truyens E et al. Perioperative management of clopidogrel therapy: the effects on in-hospital cardiac morbidity in older patients with hip fractures. Br J Anaesth 2011; 107: 911-915.

24. Plavix $75 \mathrm{mg}$ Tablets. Electronic Medicines Compendium.http://www.medicines .org.uk/emc/medicine/24207 (cited September 2015).

25. Cox G, Talbot C, Topp K, Templeton P. Clopidogrel and proximal femoral fractures: does timing of surgery affect blood loss and length of admission? A preliminary study prior to multicenter trial. Eur J Trauma Emerg Surg 2009; 35: 291-295. 